\title{
Wideband Measurements of Ice Sheet Attenuation and Basal Scattering
}

\author{
John D. Paden, Student Member, IEEE, Christopher T. Allen, Senior Member, IEEE, \\ Sivaprasad Gogineni, Fellow, IEEE, Kenneth C. Jezek, Dorthe Dahl-Jensen, and Lars B. Larsen
}

\begin{abstract}
We are developing a multifrequency multistatic synthetic aperture radar (SAR) for determining polar ice sheet basal conditions. To obtain data for designing and optimizing radar performance, we performed field measurements with a network-analyzer-based system during the 2003 field season at the North Greenland Ice Core Project camp (75.1 N and 42.3 W). From the measurements, we determine the ice sheet complex transfer function over the frequency range from 110-500 $\mathrm{MHz}$ by deconvolving out the system transfer function. Over this frequency range, we observe an increase in total loss of $8 \pm 2.5 \mathrm{~dB}$ using a linear regression to the log-scale data. With the ice sheet transfer function and an ice extinction model, we estimate the return loss from the basal surface to be approximately $37 \mathrm{~dB}$. These measurements have broad applicability to interpreting radar-sounding data, which are widely used in glaciological studies of the polar ice sheets. These data have also been used in the link budget for the design considerations of the multifrequency multistatic SAR system.
\end{abstract}

Index Terms-Ice, ultrahigh frequency (UHF) measurements, very high frequency (VHF) measurements.

\section{INTRODUCTION}

$\mathbf{T}$ HE Polar Radar for Ice Sheet Measurements (PRISM) project [1] is building a suite of sensors to determine ice sheet characteristics. One of the sensors, a multistatic multifrequency synthetic aperture radar (SAR) will image the ice-bed interface to determine basal conditions. The radar will operate over a wide frequency range to differentiate between various basal properties. The basal conditions of primary interest are wetness and roughness-both critical to modeling ice flow.

In the summer of 2003, we performed field experiments at the North Greenland Ice Core Project (NGRIP) camp. With these experiments at NGRIP, we sought to make continuous in situ measurements of radio-frequency $(\mathrm{RF})$ attenuation and provide estimates of the basal scattering characteristics from 110-500 MHz. RF attenuation and basal scattering properties are needed for the design of the radar system. All other studies of basal

Manuscript received September 6, 2004, 2004; revised November 2, 2004. This work was supported in part by the National Science Foundation under Grant OPP-0122520 and in part by the National Aeronautics and Space Administration under Grant NGT5-30449. The Danish Research Council runs the North Greenland Ice Core Project camp that supplied logistical support for these measurements.

J. D. Paden, C. T. Allen, and S. Gogineni are with the Department of Electrical Engineering and Computer Science, University of Kansas, Lawrence, KS 66045 USA (e-mail: paden@ittc.ku.edu).

K. C. Jezek is with the Byrd Polar Research Center, The Ohio State University, Columbus, OH 43210 USA.

D. Dahl-Jensen and L. B. Larsen are with the Niels Bohr Institute, Glaciology, University of Copenhagen, DK-2100 Copenhagen, Denmark.

Digital Object Identifier 10.1109/LGRS.2004.842474

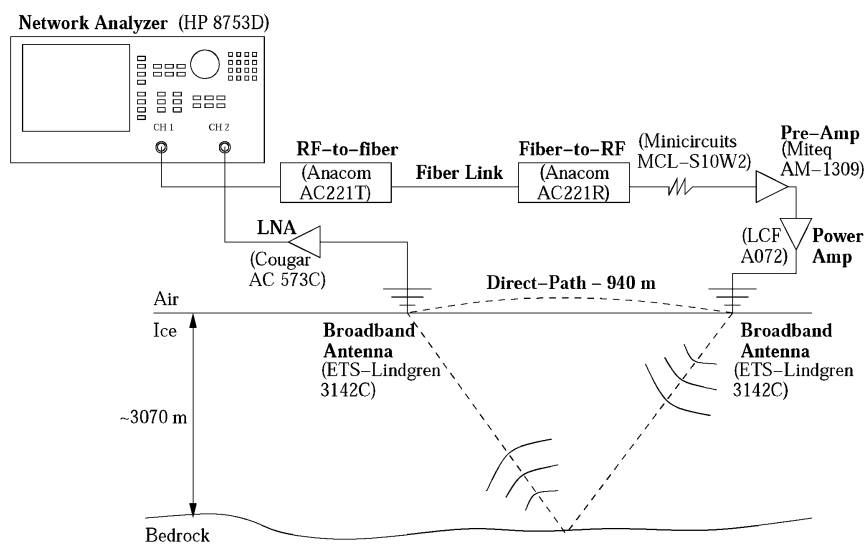

Fig. 1. Network analyzer based system used to measure the basal echo.

properties were conducted with narrowband systems [2], [3] or only at selected frequencies [4].

Our system utilizes a calibrated network analyzer and gain-calibrated antennas for step-frequency radar measurements. We deconvolve system effects and employ the radar range equation for a planar interface, and temperature, density, and conductivity profiles to determine the basal return loss.

\section{Measurement System}

We used the system shown in Fig. 1 to measure the combined system and ice sheet complex transfer function. The measurement system was divided onto two sleds separated by $940 \mathrm{~m}$, with a fiber link connection between the two sleds. One sled held the RF-to-fiber transceiver, low-noise amplifier (LNA), receive antenna, network analyzer, and computer. The other sled held the fiber-to-RF transceiver, preamplifier, power amplifier, and transmit antenna. The network analyzer generated and received the transmit chirp. The computer automated and recorded the measurements.

The maximum number of frequency points measurable by the network analyzer in a single sweep is 1601 . Our bandwidth, 390 $\mathrm{MHz}$, and range, $18-\mathrm{km}$ free-space equivalent, necessitated dividing the bandwidth into subbands. The network analyzer generated and recorded narrowband chirps on each subband and outputted the complex transfer function. We then concatenated the subband measurements in the frequency domain to form a single measurement. Separating the measurement into subbands enabled us to adjust the analyzer transmit power for each subband. This is important because the power amplifier's maximum output power is constant, but with approximately $11 \mathrm{~dB}$ of gain fluctuations over the band of interest; hence, different input 
TABLE I

ANTENNA 3-DB BEAMWIDTH

\begin{tabular}{lll}
\hline Frequency (MHz) & E-plane (degrees) & H-plane (degrees) \\
\hline 100 & 78 & 244 \\
200 & 76 & 184 \\
300 & 55 & 102 \\
400 & 82 & 140 \\
500 & 79 & 158 \\
\hline
\end{tabular}

TABLE II

NETWORK ANALYZER PARAMETERS

\begin{tabular}{ll}
\hline \multicolumn{1}{c}{ Parameter } & \multicolumn{1}{c}{ Value } \\
\hline Model & Hewlett Packard 8753D \\
Sweep Time & 16.7 seconds per subband \\
IF Bandwidth & $100 \mathrm{~Hz}$ \\
Noise Figure (with LNA) & $41 \mathrm{~dB}$ \\
Noise Power & $-143 \mathrm{dBW}$ \\
Dynamic Range & $110 \mathrm{~dB}$ \\
Number of Points & 1601 per subband (43333 points total) \\
Number of Averages & 1 \\
Chop A/B & Off \\
Power Level & -1.3 to $10 \mathrm{dBm}$ \\
Start Frequency & $50+\mathrm{N}^{*} 14.4 \mathrm{MHz}$ \\
Stop Frequency & Start Frequency $+14.4 \mathrm{MHz}$ \\
\hline
\end{tabular}

drive levels are needed to produce a constant output power level at all frequencies.

The large separation between the transmit and receive antennas lowered the direct antenna coupling. This in turn lowered the dynamic range requirement of the measurement, and this was needed due to limitations of the network analyzer (e.g., a receiver blanking switch was not a viable option). The fiber link provided a low-loss and lightweight link between the sleds.

The power amplifier was connected to a hybrid bowtie and log-periodic antenna. An identical antenna received the directpath signal and the bottom echo (internal reflection layers were also observed). The antennas were mounted $1.75 \mathrm{~m}$ in the air, horizontally polarized, and pointed toward the specular point on the bedrock. The antenna return loss is more than $10 \mathrm{~dB}$, and its 3-dB beamwidths are given in Table I. After the antenna received the signal, it was amplified by a low-noise amplifier and passed to the network analyzer.

With the exception of the antennas, we measured the complex transfer function of individual components to determine the overall transfer function. The manufacturer provided the calibrated gain curves for each antenna. The reverse isolation and return losses of each device are such that the largest reflection between devices is less than $14 \mathrm{~dB}$.

The parameters of the network analyzer are given in Table II. The intermediate frequency (IF) bandwidth was set to $100 \mathrm{~Hz}$ for better harmonic rejection [5]. The loop sensitivity of the system is the transmitter power $(20 \mathrm{dBW})$ multiplied by the compression gain $\left(10 \log _{10}(43333)=46 \mathrm{~dB}\right)$ and then divided by the noise power ( $-143 \mathrm{dBW}$ ), which comes to $209 \mathrm{~dB}$. The signal-to-noise ratio of the bedrock echo is $35 \mathrm{~dB}$, and the total signal loss in the bedrock echo is $173 \mathrm{~dB}$, indicating a measured loop sensitivity of $208 \mathrm{~dB}$.

To test the system, we separated the antennas by $8.92 \mathrm{~m}$ and pointed them directly at each other at a height of $3.2 \mathrm{~m} ; 56 \mathrm{~dB}$ of attenuation padding was added to keep the system from saturating. Using a two-ray reflection model for horizontally polarized waves, an estimated RMS surface height of $15 \mathrm{~cm}$, and

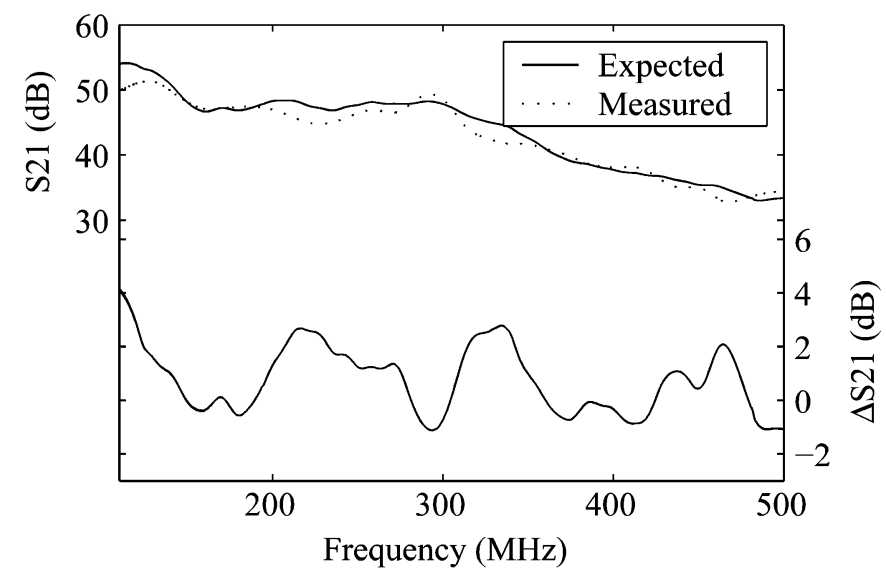

Fig. 2. Expected and measured results from system test.

snow dielectric of 1.6, we predict the magnitude of the response. The results are shown in Fig. 2. The error is between -1 and $4 \mathrm{~dB}$. There are two sources of error: inaccurate system model and inaccurate two-ray propagation model. The system model does not account for reflections between devices. This could be improved by adding padding before and after the power amplifier. The two-ray propagation model does not account for clutter from the surrounding equipment and internal reflections from the ice sheet.

The bias in the measurement of $1.5 \mathrm{~dB}$ is added into the system transfer function. Given this, the test provides an error estimate of $\pm 2.5 \mathrm{~dB}$ on our final results. Also, the antenna manufacturer does not provide the antenna phase response. For this reason, we use the phase response measured from this test in our system transfer function.

\section{ICE SHEET RF EXTINCTION}

Dielectric absorption, volume scattering from air bubbles, depolarization due to the ice birefringence, and reflections from internal layering contribute to the extinction of a radio wave as it propagates through the ice sheet. Dielectric absorption is the primary cause of signal extinction. Absorption in pure ice at radio frequencies comes from the high-frequency tail of the Debye-relaxation in the kilohertz region and the low-frequency tail of the infrared absorption bands [6]. The attenuation is controlled by the dielectric constant, which we represent by $\varepsilon=\left(\varepsilon^{\prime}-j \varepsilon^{\prime \prime}\right) \varepsilon_{0}$, where $\varepsilon_{0}$ is the permittivity of free space, and $\varepsilon^{\prime}$ and $\varepsilon^{\prime \prime}$ are the real and imaginary parts, respectively, of the relative permittivity. The justification of the following permittivity model is described in detail elsewhere [7]. For pure ice, $\varepsilon$ can be modeled by

$$
\varepsilon^{\prime}=3.1884+0.00091(T-273.15)
$$

and

$$
\varepsilon^{\prime \prime}=\frac{A_{m}}{f_{G}}+B_{m} f_{G}^{C_{m}} .
$$

Equation (1) is based on Matzler and Wegmüller's work [6], where $T$ is the temperature in kelvin. The first term of (2) accounts for Debye-relaxation loss, and the second term accounts for infrared absorption loss. The frequency $f_{G}$ is in gigahertz. 
The coefficients $A_{m}, B_{m}$, and $C_{m}$ are temperature-dependent empirical constants and are tabulated in [8], although the form of (2) is originally from [6]. Spline interpolation with not-a-knot end conditions is used to fill in values at different temperatures.

The acid and salt impurities in the ice contribute significantly to the ice permittivity. To determine their contribution, we use the calibrated dielectric profile (DEP) from the GRIP ice core. The DEP and electrical conductivity measurements from the NGRIP ice core, which provide information about impurities, have not been calibrated. For our analysis, then, we assume that the GRIP ice core, located $324 \mathrm{~km}$ south of NGRIP, is representative of the conductivity profile at NGRIP. The DEP-derived conductivity profile from GRIP gives the high-frequency limit of conductivity measured at low frequency (LF) and corrected to $258 \mathrm{~K}$ [9]. Fujita et al. [7] suggest that the high-frequency limit conductivity is valid at our frequencies, since the molar conductivity does not change from LF to ultrahigh frequency (UHF).

The DEP-derived conductivity is sensitive to the Debye-relaxation and impurities, but not infrared absorption. Therefore, we use the conductivity profile only to estimate the impurity component of conductivity. Because of this, we need to subtract off the LF pure ice conductivity. Using the single-frequency Debye model [9] suggests this value to be $9 \mu \mathrm{S} \cdot \mathrm{m}^{-1}$ at $258 \mathrm{~K}$. Due to errors in the conductivity profile, there are a few points in the profile where the total conductivity is measured to be less than $9 \mu \mathrm{S} \cdot \mathrm{m}^{-1}$, leading to a negative conductivity due to impurities. We set the impurity component of the conductivity to zero in these cases.

To determine the conductivity due to impurities at other temperatures, we use an Arrenhius model (e.g., [10, eq. (3)])

$$
\sigma_{\infty}=\sigma_{\infty, 258} \exp \left(\frac{E}{R}\left(\frac{1}{T_{258}}-\frac{1}{T}\right)\right)
$$

where $\sigma_{\infty, 258}$ is the impurity component of the conductivity from the profile, $E=0.22 \mathrm{eV}\left(21217 \mathrm{~J} \cdot \mathrm{mol}^{-1}\right)$ is the activation energy suggested by Wolff et al. [9], $R=8.3144 \mathrm{~J} \cdot \mathrm{mol}^{-1} \cdot \mathrm{K}^{-1}$ is the universal gas constant, $T$ is the desired temperature in kelvin, and $T_{258}=258 \mathrm{~K}$ is the temperature that the conductivity profile is given for. The conductivity is related to the imaginary part of the permittivity by $\varepsilon^{\prime \prime}=\left(\sigma_{\infty}\right) /\left(2 \pi f \varepsilon_{0}\right)$ where $f$ is the frequency in hertz.

The above equations (1)-(3) are for solid ice with a density of $d_{\text {ice }}=917 \mathrm{~kg} \cdot \mathrm{m}^{-3}$. However, the density of the ice sheet is a function of depth in the firn/ice transition region. To account for a density of $d$, we scale the real part of the permittivity by [11]

$$
D^{\prime}=\frac{1+1.7 d+0.7 d^{2}}{1+1.7 d_{\text {ice }}+0.7 d_{\text {ice }}^{2}}
$$

and the imaginary part of the permittivity by [11]

$$
D^{\prime \prime}=\frac{0.52 d+0.62 d^{2}}{0.52 d_{\text {ice }}+0.62 d_{\text {ice }}^{2}} .
$$

To determine the total loss $L$ as the radio wave propagates to and from the bedrock, we discretize the temperature, density, and conductivity profiles shown in Fig. 3 and find the attenuation through each discrete layer. In each case, the initial and final

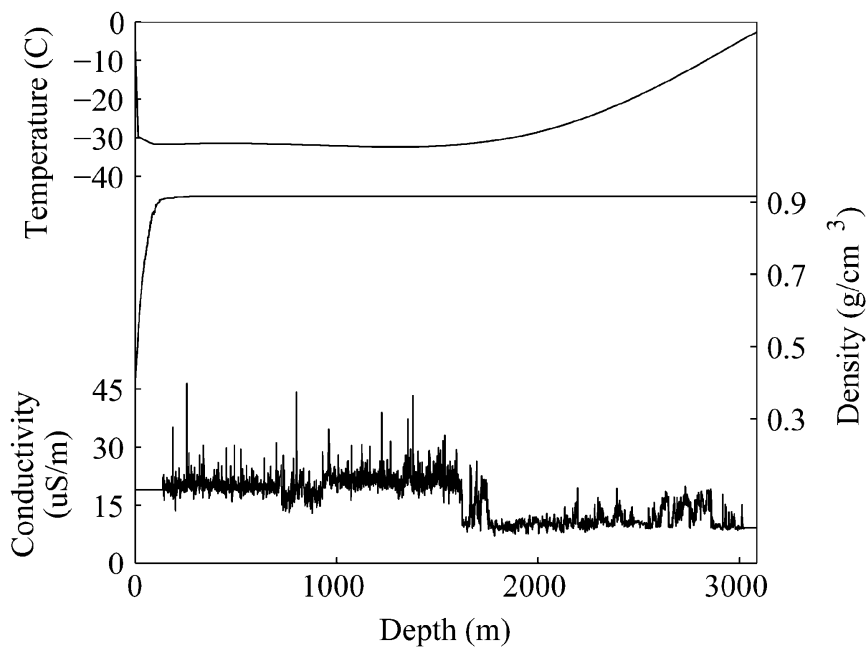

Fig. 3. Temperature and density profiles from the NGRIP ice core and conductivity profile from the GRIP ice core [9].

parts of the profiles had to be extrapolated to the top or bottom of the ice sheet. For example, the temperature profile is only known to $2987 \mathrm{~m}$, and the final $83 \mathrm{~m}$ are simulated by linear extrapolation to the base. $L$ is a weak function of frequency and increases from $49.8-52.7 \mathrm{~dB}$ over the frequency range of $110-500 \mathrm{MHz}$.

The three other causes of RF extinction are arguably very small. Volume scattering from air bubbles has been shown to be negligible at our frequencies of operation [12]. Depolarization from ice birefringence has been shown to cause losses as large as $10 \mathrm{~dB}$ [13]. However, there are two reasons to suspect that depolarization did not affect our results significantly. The first is that NGRIP is located along the ice sheet divide, and the ice flow is only $1.33 \mathrm{~m}$ per year [14]. So, it is unlikely that there is a strong horizontal preference in the ice crystal fabric [15]. The second is that a ground-based version of the University of Kansas 150-MHz coherent radar depth sounder (CoRDS) [16] mapped an ice thickness grid over NGRIP during the same field season, and no preference in antenna orientation was found (transmit and receive antennas were always copolarized, however). Unfortunately, we did not take cross-polarization measurements to determine depolarization, so the extent to which it affected our measurements is unknown.

Changes in the ice sheet permittivity give rise to internal reflections [7]. The ice sheet can be approximated by a layered medium with permittivity determined by the density, impurities, and crystal orientations of each layer. We consider briefly the effect of power loss from internal layer reflections. Using the temperature and conductivity profile shown in Fig. 3 and high-resolution snow pit density data down to $15 \mathrm{~m}$ from the Greenland Ice Sheet Project 2 (GISP2) region [17], we determine the permittivity using the method described above. The NGRIP density profile was not used for this analysis because its resolution is not fine enough. With a reflection analysis using effective wave impedances for layered media with planar interfaces [18], we simulate the power reflected from the first $15 \mathrm{~m}$ of ice. The two-way transmission loss due to the reflections is $0.21 \mathrm{~dB}$. Assuming a worst case situation where the reflections are of similar magnitude for the first $100 \mathrm{~m}$ of ice gives a transmission loss of $1.40 \mathrm{~dB}$. 




Fig. 4. Bedrock echo return and range-gating limits (vertical dashed lines). An approximate depth axis is used.

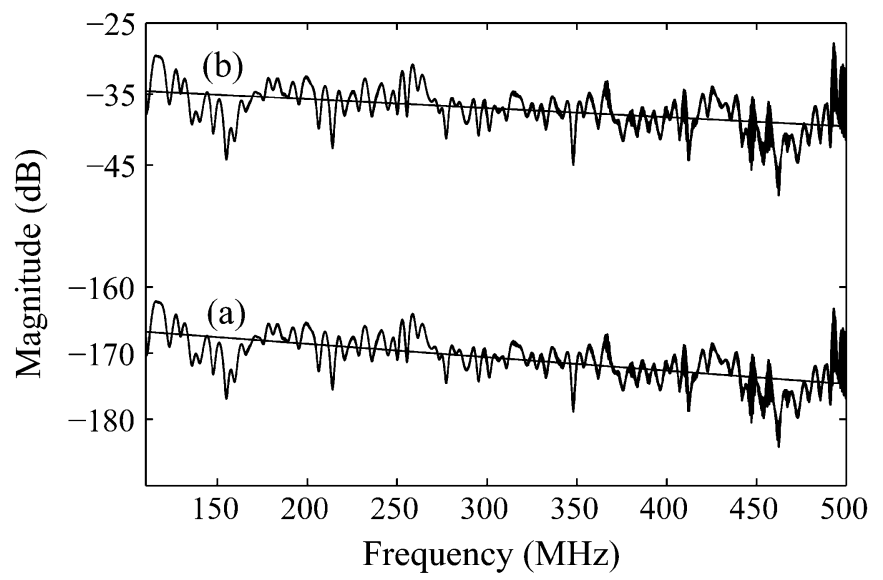

Fig. 5. (a) Total transfer function of the ice sheet. (b) Basal power reflection coefficient.

\section{Discussion AND Results}

The strength of the basal echo is derived from a matched filter (Fig. 4). To determine the transfer function of the basal echo, we first deconvolve the system transfer function out of the measured signal. We then range-gate the basal echo (dashed lines in Fig. 4) to obtain the transfer function (Fig. 5). The estimated transfer function is the incoherent average of 13 measurements made by moving the receive antenna in a straight line in $1-\mathrm{m}$ increments, thereby reducing the uncertainties of individual measurements. The oscillations in Fig. 5(a) arise from two sources. One is the bedrock roughness, which will give rise to an oscillatory frequency response. Second, the deconvolution does not remove reflections in the system, likewise leading to oscillations in the frequency response.

A linear regression of the data in Fig. 5(a) is drawn with the data and shows that the loss increases from 166.8 to $174.6 \mathrm{~dB}$ over the frequency range of $110-500 \mathrm{MHz}$. The transfer function verifies the slight frequency dependence of the ice sheet. It also shows the feasibility of a wideband or a UHF narrowband system to measure the bedrock echo in thick cold ice. Bogorodsky also collected estimates of total loss over this frequency range and reports weak frequency dependence [4].

Next, we use the specular radar equation to determine the power reflection coefficient of the ice/bedrock interface, $\Gamma$. We neglect the effects of volume scattering, depolarization, and internal reflections so that the specular radar equation is

$$
\frac{P_{R}}{P_{T}}=\left(\frac{G \lambda n_{\text {ice }}}{4 \pi(2 R)}\right)^{2} \frac{T_{\mathrm{AI}} \Gamma}{L}
$$

where $\left(P_{R}\right) /\left(P_{T}\right)$ is the ratio of power received to power transmitted and is measured by the network analyzer, $G$ is the gain of each antenna, $\lambda$ is the free-space wavelength, $n_{\text {ice }}=1.78$ is an approximate index of refraction of ice and accounts for the focusing gain in ice, $R \approx \sqrt{3070^{2}+470^{2}}=3106 \mathrm{~m}$ is the distance to the specular point, and $T_{\mathrm{AI}}=-0.8 \mathrm{~dB}$ is the two-way power transmission coefficient through the air-ice interface at $18^{\circ}$ incidence.

To find the basal power reflection coefficient, we solve (6) for $\Gamma$. However, $G$ and $\lambda$ are already removed during the deconvolution process. The estimated power reflection coefficient $\Gamma$ is plotted as a function of frequency in Fig. 5(b). Because the ice loss $L$ is removed, the frequency dependence of $\Gamma$ is only $5 \mathrm{~dB}$, decreasing from -34.7 to $-39.7 \mathrm{~dB}$. This frequency dependence is slight enough that it could be caused by errors in the calculation of $\Gamma$ or a feature specific to this particular location and sensor geometry.

Water was found at the base of the NGRIP borehole in 2003, and frozen reddish mud was found at the base in 2004. Assuming a high clay content and no water, the return loss determined by the Fresnel reflection coefficient is between 13-33 $\mathrm{dB}$ according to data tabulated in [13]. The red content could be due to iron oxides, which would indicate the smaller return loss is more accurate. If there is water, then the bedrock return loss will also be lower.

These expected return losses are lower than what was measured. Possible sources of error in the return loss are: 1) that the salt and acid impurities are different at NGRIP than GRIP; 2) we underestimate loss from volume scattering, internal reflections, and depolarization; 3) the specular reflection model is inadequate due to rough surface scattering and volume scattering; and 4 ) the ice loss model underestimates the attenuation coefficient.

Since NGRIP's base is thought to have small slopes—around $2 \%$ based on the CoRDS measurements - the scattering losses should be small at NGRIP. Also, the bedrock signal stays coherent over a Fresnel zone, indicating a specular reflection. This implies that the specular radar range equation accurately estimates the bedrock reflection coefficient. There could still be a small-scale roughness, which exponentially weights the specular radar equation.

To see how this ice model compares with other datasets of ice permittivity, we look at Westphal's data, quoted in [13], and Johari's data [19], which have been tabulated by Matsuoka for use in (2) [8]. Since Westphal's ice sample probably contains impurities, as it was taken from Tuto Tunnel, Greenland, we assume that the loss from impurities is included in his data. Our model estimates $L$ to be $\sim 50 \mathrm{~dB}$, while using these two datasets we estimate $L$ to be $\sim 87 \mathrm{~dB}$ (Westphal) and $\sim 93 \mathrm{~dB}$ (Johari). These latter values of $L$ indicate that the bedrock reflectivity is 0 and $6 \mathrm{~dB}$, respectively. Both these values are physically unrealizable and suggest that these datasets overestimate loss. The 
imaginary part of relative permittivity, which is very difficult to measure for loss-low materials, may have been overestimated in the earlier measurements.

The combination of our wideband radar measurements at the NGRIP site with in situ physical properties measured at ice-core drilling sites provides new insights into the propagation of electromagnetic waves in ice sheets. However, additional measurements are needed both for designing imaging radar systems and interpreting data from these systems, including: updated VHF measurements of ice attenuation, cross-polarization measurements, and finally, the collection and analysis of bedrock echoes over a much larger region than was possible with this data collection system.

\section{ACKNOWLEDGMENT}

The authors thank all those who helped with and supported the field experiments, especially A. Agah, T. Akins, D. Braaten, P. Kanagaratnam, and H. Rufli. The authors also thank the reviewers for their valuable suggestions.

\section{REFERENCES}

[1] S. Gogineni, G. Prescott, D. Braaten, C. Allen, and K. Jezek, "Polar radar for ice sheet measurements," in Proc. IGARSS, Toulouse, France, Jul. 2003, pp. 1607-9.

[2] C. Bentley, N. Lord, and C. Liu, "Radar reflections reveal a wet bed beneath stagnant ice stream $\mathrm{C}$ and a frozen bed beneath ridge $\mathrm{BC}$, West Antarctica," J. Glaciol., vol. 44, no. 146, pp. 149-156, 1998.

[3] H. Ohmae, F. Nishio, and S. Mae, "Distribution of reflected power from the bed by radio echo-sounding in the shirase glacier drainage area, East Dronning Maud Land, Antarctica," Ann. Glaciol., vol. 12, pp. 124-126, 1989.

[4] V. Bogorodsky, C. Bentley, and P. Gudmandsen, Radioglaciology. Amsterdam, The Netherlands: Reidel , 1985, pp. $122-124$.

[5] Hewlett Packard, Hewlett Packard 8753D Network Analyzer User Guide. Palo Alto, CA: Hewlett Packard, 1995, pp. 6-37.
[6] C. Mätzler and U. Wegmüller, "Dielectric properties of fresh-water ice at microwave frequencies," J. Phys. D: Appl. Phys., vol. 20, pp. 1623-1630, 1987.

[7] S. Fujita, T. Matsuoka, T. Ishida, K. Matsuoka, and S. Mae, "A summary of the complex dielectric permittivity of ice in the megahertz range and its applications for radar sounding of polar ice sheets," in Physics of Ice Core Records, T. Hondoh, Ed. Sapporo, Japan: Hokkaido Univ. Press, 2000, pp. 185-212.

[8] T. Matsuoka, S. Fujita, and S. Mae, "Effect of temperature on dielectric properties of ice in the range 5-39 GHz," J. Appl. Phys., vol. 80, no. 10, pp. 5884-5890, Nov. 15, 1996.

[9] E. Wolff, J. Moore, H. Clausen, C. Hammer, J. Kipfstuhl, and K. Fuhrer, "Long-term changes in the acid and salt concentrations of the Greenland ice core project ice core from electrical stratigraphy," J. Geophys. Res., vol. 100, no. D8, pp. 16249-16263, Aug. 20, 1995. Data archived at the World Data Center for Paleoclimatology.

[10] H. Corr, J. Moore, and K. Nicholls, "Radar absorption due to impurities in Antarctic ice," Geophys. Res. Lett., vol. 20, no. 11, pp. 1071-1074, Jun. 7, 1993.

[11] M. Tiuri, A. Sihvola, E. Nyfors, and M. Hallikaiken, "The complex dielectric constant of snow at microwave frequencies," IEEE J. Oceanic Eng., vol. OE-9, no. 5, pp. 377-382, Dec. 1984.

[12] Q. Robin, S. Evans, and J. Bailey, "Interpretation of radio echo sounding in polar ice sheets," Phil. Trans. R. Soc. London., Series A, Math. Phys. Sci., vol. 265, no. 1166, pp. 437-505, Dec. 18, 1969.

[13] G. Jiracek, "Radio sounding of Antarctic ice," Univ. Wisconsin, Madison, The Univ. Wisconsin Geophys. Polar Res. Center Res. Rep. Series 67-1, Nov. 1967.

[14] D. Dahl-Jensen, N. Gundestrup, S. Gogineni, and H. Miller, "Basal melt at NorthGRIP modeled from borehole, ice-core, and radio-echo sounder observations," Ann. Glaciol., vol. 37, pp. 207-212, 2003.

[15] S. Fujita and S. Mae, "Relation between ice sheet internal radio-echo reflections and ice fabric at Mizuho Station, Antarctica," Ann. Glaciol., vol. 17 , pp. 269-275, 1993

[16] S. Gogineni et al., "Coherent radar ice thickness measurements over the Greenland ice sheet," J. Geophys. Res., vol. 106, no. D24, pp. 33 761-72, Dec. 27, 2001.

[17] J. Bolzan and M. Strobel, "Accumulation-rate variations around summit, Greenland," J. Glaciol., vol. 40, pp. 56-66, 1994. Data archived at the World Data Center for Paleoclimatology.

[18] K. Demarest, Engineering Electromagnetics. Upper Saddle River, NJ: Prentice-Hall, 1998.

[19] G. P. Johari and P. A. Charette, "The permittivity and attenuation in polycrystalline and single-crystal ice Ih at 35 and $60 \mathrm{MHz}$," J. Glaciol., vol. 14, no. 71, pp. 293-303, 1975. 